\title{
ON WELL-BOUNDED OPERATORS OF CLASS $\Gamma$
}

\author{
by ADNAN A. S. JIBRIL
}

(Received 30 October, 1980)

1. Let $T$ be a linear operator acting in a Banach space $X$. It has been shown by Smart [5] and Ringrose [3] that, if $X$ is reflexive, then $T$ is well-bounded if and only if it may be expressed in the form

$$
T=\int \lambda d E(\lambda)
$$

where $\{E(\lambda)\}$ is a suitable family of projections in $X$ and the integral exists as the strong limit of Riemann sums.

In [4], Ringrose considered the extension of this, and related results, to the nonreflexive case. The theory obtained is less staisfactory, in that it is necessary to work with projections acting in the dual space $X^{*}$ rather than in $X$ itself, and those projections are no longer (in general) uniquely determined.

Turner [6] considered the case where the projections $E(\lambda)$ are acting in $L(X)$ and obtained a class of operators each of which is called a scalar-type decomposable operator of class $\Gamma$.

In this paper we define the class of well-bounded operators of class $\Gamma$ and we show that this is equivalent to the class of operators defined by Turner.

This paper is a part of a dissertation presented for the degree of Doctor of Philosophy in Glasgow University. The author wishes to express his gratitude to Dr. H. R. Dowson for his guidance and encouragement.

2. Notation. Throughout $X$ is a complex Banach space with dual space $X^{*}$. We write $\langle x, y\rangle$ for the value of the functional $y$ in $X^{*}$ at the point $x$ of $X$. The Banach algebra of bounded linear operators on $X$ is denoted by $L(X)$. The spectrum of $T$, in $L(X)$, is denoted by $\sigma(T)$. We use $[a, b]$ to denote a compact interval of the real line $\mathbf{R}$. The symbol $\Gamma$ is used to denote a total subset of $X^{*}$; that is if $x \in X$, and $\langle x, y\rangle=0$, for all $y \in \Gamma$, then $x=0$. As usual the symbol $C(K)$ is used to denote the algebra of all continuous, complex-valued functions on $K$, and $I$ is used to denote the identity operator in $L(X)$.

\section{Scalar-type decomposable operators of class $\Gamma$.}

Definitions 3.1. Let $\{E(t): t \in \mathbf{R}\}$ be a family of projections in $L(X)$ with the following properties:

(1) $E(s)=0(s<a), E(s)=I(s \geq b)$;

(2) $E(s) E(t)=E(t) E(s)=E(s)(s \leq t)$;

(3) there is a real constant $k$ such that

$$
\|E(s)\| \leq k \quad(s \in \mathbf{R})
$$

Glasgow Math. J. 24 (1983) 1-5. 
(4) the function $s \rightarrow\langle E(s) x, y\rangle$ is Lebesgue measurable $(x \in X, y \in \Gamma)$;

(5) if $x \in X, y \in \Gamma, a \leq s<b$, and if the function

$$
t \rightarrow \int_{a}^{t}\langle E(u) x, y\rangle d u
$$

is right differentiable at $s$, then the right derivative at $s$ is $\langle E(s) x, y\rangle$;

(6) for each $x \in X$, the map

$$
y \rightarrow\langle E(\cdot) x, y\rangle
$$

from $\Gamma$ into $L^{\infty}(a, b)$ is continuous when $\Gamma$ is given the $\Gamma$-topology and $L^{\infty}(a, b)$ is given its weak ${ }^{*}$-topology (as the dual of $L^{1}(a, b)$ );

(7) if $x, y$ in $X$ and $z \in \Gamma$ are such that

$$
\langle y, z\rangle=\int_{a}^{b}\langle E(t) x, z\rangle d t ;
$$

then for almost all $u$ in $[a, b]$ we have

$$
\langle E(u) y, z\rangle=\int_{a}^{b}\langle E(t) E(u) x, z\rangle d t .
$$

Then $\{E(t): t \in R\}$ is called a decomposition of the identity for $X$ of class $\Gamma$.

It is a consequence of $(6)$ and $([2$, Theorem 3.9, p. 421]) that there exists a unique operator $T$ in $L(X)$ such that

$$
\langle T x, z\rangle=\langle x, z\rangle-\int_{a}^{b}\langle E(t) x, z\rangle d t \quad(x \in X, z \in \Gamma) .
$$

$\{E(t): t \in \mathbf{R}\}$ is called an $S$-decomposition of the identity of class $\Gamma$ for $T$, and $T$ is called a scalar-type decomposable operator of class $\Gamma$.

The above definition is due to Turner ([6, Definition 3.4, p. 524]). We call an operator $T$, in $L(X)$, which satisfies conditions (1)-(6) above a well-bounded operator of class $\Gamma$.

PROPOSITION 3.2. For a well-bounded operator of class $\Gamma$, the following two conditions are equivalent.

(i) If $x, y$ in $X$ and $z$ in $\Gamma$ are such that

$$
\langle y, z\rangle=\int_{a}^{b}\langle E(t) x, z\rangle d t,
$$

then for almost all $u$ in $[a, b]$ we have

$$
\langle E(u) y, z\rangle=\int_{a}^{b}\langle E(t) E(u) x, z\rangle d t .
$$

(ii) For each $x$ in $X$ and $z$ in $\Gamma$,

$$
\langle E(u) T x, z\rangle=\langle T E(u) x, z\rangle
$$

for almost all $u$ in $[a, b]$. 
Proof. Let $x, y$ in $X$ and let $z$ in $\Gamma$. Then, by Definition 3.1, we have

$$
\langle T x, z\rangle=\langle x, z\rangle-\int_{a}^{b}\langle E(t) x, z\rangle d t
$$

which implies that

$$
\langle x-T x, z\rangle=\int_{a}^{b}\langle E(t) x, z\rangle d t
$$

Putting $y=x-T x$, we get

$$
\langle y, z\rangle=\int_{a}^{b}\langle E(t) x, z\rangle d t
$$

Hence, supposing that condition (i) above is true, we get

$$
\langle E(u) y, z\rangle=\int_{a}^{b}\langle E(t) E(u) x, z\rangle d t
$$

for almost all $u$ in $[a, b]$. Now by replacing $x$ by $E(u) x$ in (3.2.1), we obtain

$$
\langle E(u) x-T E(u) x, z\rangle=\int_{a}^{b}\langle E(t) E(u) x, z\rangle d t
$$

Hence,

$$
\langle E(u) y, z\rangle=\langle E(u) x-T E(u) x, z\rangle .
$$

Now, substituting in (3.2.3) $y=x-T x$, we get

$$
\langle E(u) x-E(u) T x, z\rangle=\int_{a}^{b}\langle E(t) E(u) x, z\rangle d t
$$

Comparing (3.2.3) and (3.2.4) and using (3.2.2) we conclude that

$$
\langle E(u) x-E(u) T x, z\rangle=\langle E(u) x-T E(u) x, z\rangle,
$$

which implies that

$$
\langle E(u) T x, z\rangle=\langle T E(u) x, z\rangle
$$

for almost all $u$ in $[a, b]$. Hence (i) implies (ii). Now we prove that (ii) implies (i). Suppose that (ii) holds; then $T E(u)=E(u) T$ for almost all $u$ in $[a, b]$. Thus

$$
\langle E(u) x-T E(u) x, z\rangle=\langle E(u) x-E(u) T x, z\rangle
$$

Now replacing $x$ by $E(u) x$ in (3.2.1) we get

$$
\langle E(u) x-T E(u) x, z\rangle=\int_{a}^{b}\langle E(t) E(u) x, z\rangle d t
$$


Putting $x-T x=y$ we get

$$
\langle x-T x, z\rangle=\langle y, z\rangle
$$

which implies that

$$
\langle E(u) x-E(u) T x, z\rangle=\langle E(u) y, z\rangle .
$$

Since the left hand side of (3.2.6) equals the left hand side of (3.2.7), we have

$$
\langle E(u) y, z\rangle=\int_{a}^{b}\langle E(t) E(u) x, z\rangle d t,
$$

for almost all $u$ in $[a, b]$. Hence (ii) implies (i), which completes the proof.

Theorem 3.3. Let $T \in L(X)$ be a well-bounded operator of class $\Gamma$ and let $\{E(t): t \in \mathbf{R}\}$ be a decomposition of the identity of class $\Gamma$ for $T$. Then $f(T)$ commutes with $\{E(t): t \in \mathbf{R}\}$.

Proof. The proof is similar to the proof of Theorem 3.6 (v) of [6, p. 526].

It follows from 3.2 and 3.3 that the class of all well-bounded operators of class $\Gamma$ is equivalent to the class of scalar type decomposable operator of class $\Gamma$.

TheOREM 3.4. Let $T \in L(X)$ be a well-bounded operator of class $\Gamma$ and let $\{E(t): t \in \mathbf{R}\}$ be a decomposition of the identity of class $\Gamma$ for $T$. Then $T$ is well-bounded.

Proof. The proof is similar to the proof of Theorem 3.6 (i) of [6, p. 526].

Definition 3.5. Let $S \in L(X)$. We say that $S$ possesses a $C$-operational calculus $\Psi$ if there is a bicontinuous algebra isomorphism $\Psi$ from $C(\sigma(S))$ into a subalgebra of $L(X)$ such that $\Psi\left(f_{0}\right)=I$ and $\Psi\left(f_{1}\right)=S$, where

$$
f_{0}(\lambda)=1, \quad f_{1}(\lambda)=\lambda \quad(\lambda \in \sigma(S)) .
$$

Example 3.6. Let $X=C[0,1]$. Define $S$, in $L(X)$, by

$$
(S f)(t)=t f(t) \quad(f \in X, 0 \leq t \leq 1) .
$$

Then $S$ is a well-bounded operator (see [1, p. 173]) which possesses a C-operational calculus $\Psi$ given by

$$
\Psi(g) f=g f \quad(f, g \in X) .
$$

Clearly $\sigma(S)=[0,1]$. Suppose that $P^{2}=P \in L(X)$ and $S P=P S$. Let $f_{0}(t)=1$ $(0 \leq t \leq 1)$. By the Stone-Weierstrass theorem, $P f=\left(P f_{0}\right) f$, for all $f$ in $X$, so that

$$
\left(P f_{0}\right)^{2}=P f_{0} \text {. }
$$

Thus $P=O$ or $P=I$. It follows from Definition $3.1(5)$ that $S$ is a well-bounded operator but there is no total subspace $\Gamma$ such that $S$ is well-bounded of class $\Gamma$.

\section{REFERENCES}

1. H. R. Dowson, Spectral theory of linear operators (Academic Press, London, 1978).

2. N. Dunford and J. T. Schwartz, Linear operators, part I, (Wiley-Interscience, 1958). 
3. J. R. Ringrose, On well-bounded operators. J. Austral. Math. Soc. 1 (1960), 334-343.

4. J. R. Ringrose, On well-bounded operators, II. Proc. London Math. Soc. (3) 13 (1963), 613-638.

5. D. R. Smart, Conditionally convergent spectral expansions. J. Austral. Math. Soc. 1 (1960) 319-333.

6. J. K. Turner, On well-bounded and decomposable operators. Proc. London Math. Soc. (3) 37 (1978), 521-544.

YARMOUK UNIVERSITY

IRBID-JORDAN
Present address:

University of Petroleum ANd Minerals

P.O. BOX 1950

DHAHRAN

SAUdi ARABIA 